\title{
PREVALÊNCIA DE DOADORES DE ÓRGÃOS E TECIDOS ENTRE ESTUDANTES DO ENSINO MÉDIO E SUPERIOR
}

\author{
Prevalence of organs and tissues donors among High school and college students
}

Vanessa dos Santos Silva, Bartira de Aguiar Roza, Alessandra dos Santos Silva, Janine Schirmer

\begin{abstract}
RESUMO
Mudanças na Lei 9.434/97, por meio de medida provisória e posterior alteração (Lei 2.211/2001), relacionadas aos tipos de doação, transformado-a de doação presumida (1997) para consentida (2001) podem ter colaborado, entre outros fatores, para a dificuldade de incorporação da cultura de doação em nosso país. Conhecer a opinião de estudantes é, portanto, uma condição necessária para desenvolver estratégias de educação e auxiliar na construção de legislação que represente, de fato, a moral vigente de uma população. Objetivo: investigar a prevalência de doadores de órgãos e tecidos entre estudantes do ensino médio e superior e seu conhecimento sobre o consentimento familiar como prerrogativa para a doação no Brasil. Métodos: Estudo de abordagem quantitativa, baseado na comparação entre variáveis semelhantes de duas pesquisas sobre o conhecimento de graduandos em saúde de uma comunidade universitária e estudantes do último ano do ensino médio do município de São Paulo, realizadas em períodos diferentes, 2002/2003 e 2005/2006, respectivamente. Resultados: 47,0\% (363) dos estudantes do ensino médio e 50,6\% (316) dos estudantes do ensino superior não sabiam que a doação de órgãos e tecidos no Brasil é consentida. Há evidências de que haja um maior percentual de não doadores entre os alunos de graduação e um maior percentual de doadores entre os alunos do ensino médio. Conclusão: A prevalência de doadores entre os estudantes em geral foi de 66,3\% (946), enquanto os não doadores representaram 23,1\% (329); 8,9\% (127) não sabem e 1,8\% (25) não informaram. Portanto, estratégias educacionais, e não somente informativas, precisam fazer parte do currículo da educação básica brasileira, para o desenvolvimento de uma cultura de doação no país, que subsidiaria a decisão de ser ou não doador. Essas estratégias dependerão não somente da regulamentação dos governos, mas também da vontade dos principais envolvidos nesse processo para que funcionem.
\end{abstract}

Descritores: Estudantes, Transplante, Opinião Pública, políticas, Bases de Conhecimento.

Instituição:

Universidade Federal de São Paulo - São Paulo - SP - Brasil

Correspondência:

Vanessa dos Santos Silva

Endereço: Rua Azagaia, 20A, Vila Rica, São Paulo - SP - CEP: 03912-170

Fax: (11) 6910-0526

E-mail: nessinha4ever@hotmail.com

Recebido em: 10.10.2007

Aceito em: 02.01.2008

\section{INTRODUÇÃO}

Na década de 1960 iniciou-se a atividade de transplante de órgãos e tecidos no Brasil e, em 1965, foram realizados os dois primeiros transplantes do país na cidade de São Paulo. ${ }^{1}$

Devido ao baixo sucesso dos transplantes na época citada, pouco se difundiu sobre o assunto. Após alguns anos, com o desenvolvimento tecnológico e científico, o transplante estabeleceu-se como uma opção terapêutica, sendo necessário criar regulamentações para a doação e transplante de órgãos.

Em 1997 foi promulgada a Lei $\mathrm{n}^{\circ}$ 9.434, que definiu o processo de doação/transplante no Brasil e criou o Sistema Nacional de Transplante, pela qual todo e qualquer cidadão brasileiro passava a ser doador, a menos que não houvesse seu consentimento, sendo necessário expressar sua vontade na carteira nacional de habilitação ou no registro geral (doação presumida). ${ }^{2,3}$

Devido à grande polêmica gerada por essa lei, foi criada uma medida provisória, em 6 de outubro de 1998, que acrescentou um parágrafo ao art. $4^{\circ}$ da Lei $n^{\circ} 9.434 / 97$, aonde as família passa a decidir pela doação ou não, nos casos de ausência da manifestação expressa nos documentos previstos em lei, pelo potencial doador. ${ }^{4,5}$ 
A Medida Provisória assumiu o caráter de Lei n 10.211 , em março de 2001, introduzindo o Registro Nacional de Doadores, no qual as pessoas poderiam se declarar doadoras e transformava a doação de presumida fraca para consentida, ou seja, no post mortem valeria a autorização do cônjuge ou parente, maior de idade, obedecida a linha sucessória, reta ou colateral, até o segundo grau inclusive, firmado em documento subscrito por duas testemunhas presentes à verificação da morte. ${ }^{6}$

A doação de órgãos e tecidos no Brasil, quando comparada a de outros países, apresenta menor taxa de doadores por milhão de população (pmp). Na Europa, existem cerca de 40 doadores pmp, já no Brasil há por volta de seis doadores pmp.?

Estes dados permitem refletir sobre os motivos que levam às baixas taxas de doação de órgãos e tecidos para transplantes no Brasil, tendo em vista que a doação é um ato de solidariedade, que implica em diversos conceitos preexistentes, muitos dos quais adquiridos ao longo da vida.

A falta de conhecimento ou informação sobre o processo de doação ou diagnóstico de morte encefálica, o desconhecimento da família em relação à vontade/desejo do seu parente falecido, ou a insatisfação em relação à assistência prestada, são hoje considerados fatores dificultadores para a família autorizar a doação.

Sabemos que educar é diferente de informar, sendo este, o ato ou efeito de informar, transmitir notícias; e a educação, o aperfeiçoamento das faculdades físicas intelectuais e morais do ser humano; disciplinamento, instrução, ensino. ${ }^{8}$

Ambas as estratégias podem ter impacto sobre as taxas de doação e transplante, mas, na formação de recursos humanos em saúde, a metodologia de educação andragógica deve ser utilizada para capacitar os profissionais de saúde, pois existem evidências de que essas estratégias tanto para profissionais quanto para a sociedade são fatores determinantes do sucesso ou fracasso dos programas de transplante. ${ }^{4}$

Além disso, essa capacitação focada em competências e resultados que contribuem para a melhoria contínua do processo de doação, gera cultura de segurança para as famílias doadoras, exigência fundamental para a sustentabilidade dos serviços de saúde, sinônimo de qualidade.

Estudo realizado entre médicos e enfermeiros em uma comunidade universitária da cidade de São Paulo, sobre a legislação de transplantes, mostrou que: 73\% dos profissionais conheciam parcialmente o conteúdo da lei; $42 \%$ não concordavam com a nova legislação e o número de doadores passou de 14 para $34 \% .^{5}$

Estudos semelhantes mostram que, em Uppsala, Suécia, apesar de favoráveis, apenas 50\% dos estudantes do ensino médio interessam-se em doar seus próprios órgãos e $20 \%$, os de seus pais. ${ }^{9}$

Em Seattle, EUA, ao realizar um estudo que avaliava o conhecimento e a opinião também de estudantes do ensino médio quanto ao processo de doação e transplante de órgãos e tecidos, encontrou-se que mais de $50 \%$ dos estudantes não souberam responder 13 das 16 questões da pesquisa. ${ }^{10}$

Em Torino, Itália, uma pesquisa foi realizada com o objetivo de avaliar o impacto causado por programas educacionais feitos com estudantes de ensino médio sobre o interesse destes quanto à doação, aonde 76,8\% dos estudantes responderam que são favoráveis à doação e transplante de órgãos e tecidos, e aproximadamente
98\% deles apreciaram o programa educacional e sugeriram que a experiência fosse estendida a outras escolas. ${ }^{11}$

O estudo teve por objetivo investigar a prevalência de doadores de órgãos e tecidos entre estudantes do ensino médio e superior e o conhecimento sobre o consentimento familiar como prerrogativa para a doação no Brasil. Foi submetido e aprovado pelo comitê de ética em pesquisa UNIFESP ( $\left.n^{\circ} 0024 / 05\right)$.

\section{MATERIAIS E MÉTODOS}

Estudo de abordagem quantitativa, baseado na comparação entre variáveis semelhantes de duas pesquisas sobre o conhecimento de estudantes do último ano do ensino médio, graduandos em saúde de uma comunidade universitária do município de São Paulo, realizadas em períodos diferentes, entre 2002 e 2006. . $^{12,13,14}$

A partir da unificação dos dois bancos de dados totalizou-se uma população de 1.427 estudantes (conforme Quadro abaixo) do último ano do ensino médio de escolas públicas e privadas, do município de São Paulo; e alunos da Universidade Federal de São Paulo, dos cursos de Enfermagem, Medicina, Tecnologia Oftálmica, Biomedicina e Fonoaudiologia.

Quadro Amostral

\begin{tabular}{lcc}
\hline Ano/Curso & N & $\%$ \\
\hline 2002/2003 (Ensino Universitário) & 645 & 45,2 \\
2005/2006 (Ensino Médio) & 782 & 54,8 \\
Total & 1427 & 100,0 \\
\hline
\end{tabular}

Permitindo dessa forma, a identificação de informações em relação ao conhecimento sobre a legislação brasileira de doação de órgãos e o desejo de serem doadores, que foram obtidos por meio de formulário específico.

Os dados compilados foram submetidos à análise estatística descritiva para caracterizar o perfil dos estudantes utilizando medidas de tendência central (média, mediana, desvio-padrão (DP), valor mínimo e máximo. Foram utilizadas, também, variáveis qualitativas apresentadas por meio de freqüências absolutas (N) e relativas (\%).

Para analisar as comparações entre os tipos de cursos utilizou-se o teste Qui-Quadrado, para o qual foi considerado um nível de significância de $5 \%$ (p-valor $<0,05)$.

Entre os cruzamentos onde houve evidência de associação estatística e mais do que duas categorias de resposta, foi utilizada a análise de resíduo para a identificação de quais pares de categorias correspondiam a um valor acima do esperado, ou seja, quais categorias se diferenciavam entre os grupos. Um valor de resíduo padronizado $\left(\mathrm{Z}_{\text {res }}\right)$ acima de 1.96, indica significância estatística.

As variáveis consideradas para o estudo foram:

- Variáveis qualitativas: curso, sexo, natureza do ensino médio (público ou privado), conhecimento do conceito de morte encefálica, o que é preciso para ser doador e se os estudantes eram doadores ou não;

- Variável quantitativa: idade 
Tabela 1: Porcentagem de respostas à pergunta: "para ser doador é preciso?", São Paulo, 2002 a 2006.

\begin{tabular}{|c|c|c|c|c|c|c|c|c|c|c|c|c|c|}
\hline \multirow{3}{*}{ Para ser doador é preciso: } & \multicolumn{12}{|c|}{ Curso } & \multirow{3}{*}{$\mathrm{p}$-valor } \\
\hline & \multicolumn{2}{|c|}{ Biomedicina } & \multicolumn{2}{|c|}{ Enfermagem } & \multicolumn{2}{|c|}{ Fonoaudiologia } & \multicolumn{2}{|c|}{ Medicina } & \multicolumn{2}{|c|}{ Tecnologia } & \multicolumn{2}{|c|}{ Ensino } & \\
\hline & N & $\%$ & N & $\%$ & $\mathrm{~N}$ & $\%$ & $\mathbf{N}$ & $\%$ & N & $\%$ & N & $\%$ & \\
\hline Declaração & 2 & $2,4 \%$ & 19 & $7,4 \%$ & 2 & $1,8 \%$ & 4 & $2,2 \%$ & 1 & $7,1 \%$ & 67 & $8,6 \%$ & $<0,001$ \\
\hline Registro em RG ou CNH & 41 & $\begin{array}{c}50,0 \% \\
(3,2)\end{array}$ & 92 & $\begin{array}{c}35,7 \% \\
(3,2)\end{array}$ & 58 & $\begin{array}{c}52,7 \% \\
(5,2)\end{array}$ & 92 & $50,8 \%$ & 5 & $35,7 \%$ & 195 & $24,9 \%$ & \\
\hline $\begin{array}{l}\text { Comunicação à família ou } 0 \\
\text { responsável }\end{array}$ & 25 & $30,5 \%$ & 124 & $\begin{array}{c}48,1 \% \\
(5,8)\end{array}$ & 33 & $30,0 \%$ & 65 & $35,9 \%$ & 3 & $21,4 \%$ & 216 & $27,6 \%$ & \\
\hline Todas as anteriores & 11 & $13,4 \%$ & 21 & $8,1 \%$ & 16 & $\begin{array}{c}14,5 \% \\
(2,3)\end{array}$ & 7 & $3,9 \%$ & 4 & $\begin{array}{c}28,6 \% \\
(2,7)\end{array}$ & 63 & $8,1 \%$ & \\
\hline Outras & 3 & $3,7 \%$ & 2 & $0,8 \%$ & 1 & $0,9 \%$ & 13 & $7,2 \%$ & 1 & $7,1 \%$ & 241 & $30,8 \%$ & \\
\hline
\end{tabular}

\section{RESULTADOS}

A média das idades foi de 18,9 anos com um máximo de 47 e mínimo de 16 anos; DP de $\pm 2,7$ anos, havendo uma variância de 7,3 anos. Havia uma maior prevalência de adolescentes, pois 54,8\% do total dos estudantes (1.427) eram do terceiro ano do ensino médio e tinham idades entre16 e 19 anos (89,5\%), sendo que a maioria era do sexo feminino.

Quanto ao tipo de ensino médio que os estudantes cursaram ou estavam cursando, a maioria dos graduandos (91,5\% biomedicina, 92,4\% enfermagem, 69,1\% fonoaudiologia, 92,3\% medicina), fez o ensino médio privado, com exceção dos estudantes de tecnologia oftálmica (42,8\%), enquanto que 80,9\% dos estudantes do ensino médio cursavam a rede pública.

Para a variável “Para ser doador é preciso?”, há evidências de que, dos estudantes do ensino médio, 27,6\% sabiam que a doação de órgãos e tecidos no Brasil é consentida, mas 24,9\% deles ainda confundiam o tipo de doação com presumida (na qual o doador deve expressar seu desejo em carteira de identidade ou carteira nacional de habilitação). 30,8\% deles escolheram alternativas que configuraram uma miscelânea de informações (Tabela 1).

Figura 1. Percentagem de Doadores do Ensino Universitario (2002/2003) e Médio (2005/2006)

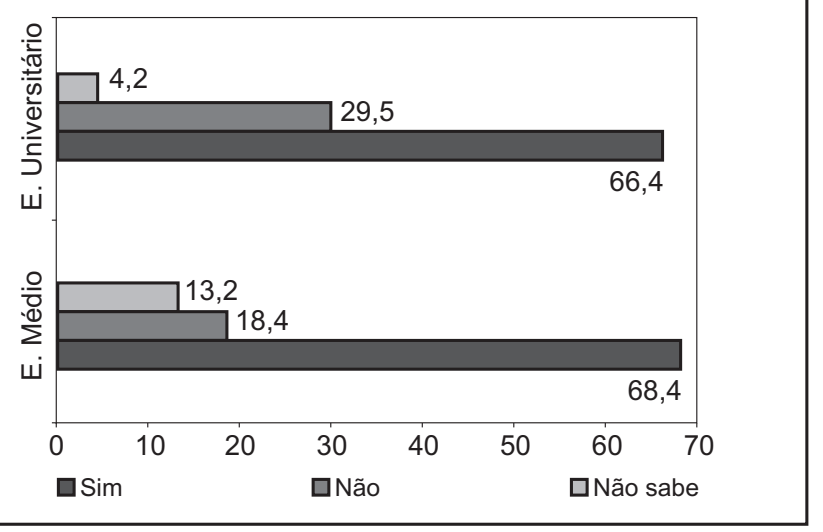

A maioria dos adolescentes e adultos jovens dos cursos na área da saúde acreditava que, para ser doador, era preciso registrar seu desejo em registro geral ou carteira nacional de habilitação, sendo $50,0 \%$ biomedicina, 35,7\% enfermagem, 52,7\% fonoaudiologia, $50,8 \%$ medicina e $35,7 \%$ tecnologia oftálmica.

Considerando a resposta ainda não sabe na pergunta se “É doador?”, por haver diferença entre os anos de 2002/2003 e 2005/2006 $(\mathrm{p}<0,05)$ e ter havido mais de duas possibilidades de resposta, foi realizada a análise de resíduo na comparação desta variável. Após a análise de resíduos foi possível constatar evidências de que: há um maior percentual de não doadores entre os alunos de 2002/2003 e um maior percentual de alunos que ainda não sabem se serão doadores entre os alunos de 2005/2006 (Figura 1).

Excluindo a resposta ainda não sabe na pergunta se "É doador?", há evidências de que há um maior percentual de não doadores entre os alunos de 2002/2003 e um maior percentual de doadores entre os alunos de 2005/2006 (Figura 2).

Com relação ao critério para ser um doador, apesar de haver indicações de que o percentual de alunos que respondem corretamente (“comunicar a família”) nos anos de 2005/2006 seja maior, não há evidências estatísticas ( $\mathrm{p}>0,05)$ de que haja diferença entre os anos.

Figura 2. Percentagem de Conhecimento sobre o critério para ser um doador de órgãos e tecidos em relação ao Ensino Universitário (2002/2003) e Médio (2005/2006).

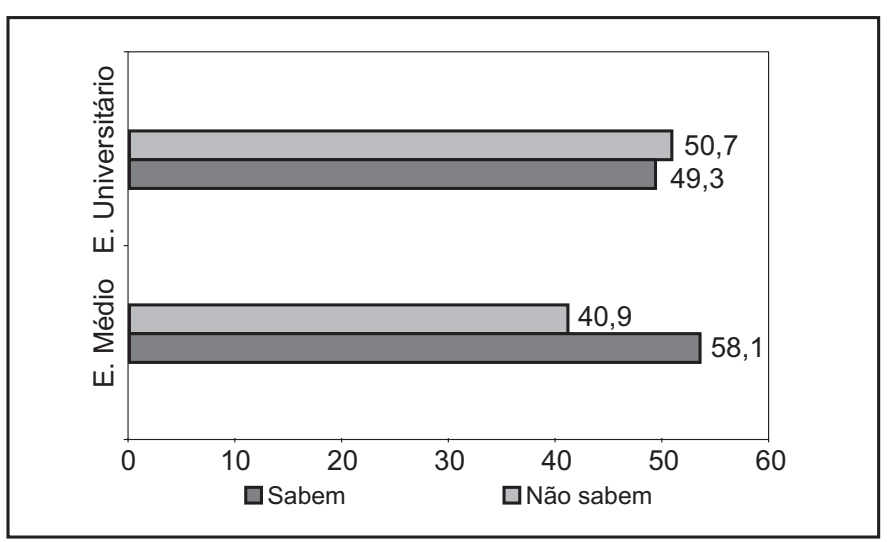




\section{DISCUSSÃO}

Agrupando-se os dados, percebe-se que não há uma diferença estatística entre a natureza do ensino médio (público e privado). Provavelmente, esta análise pode ter sido prejudicada devido a um maior número de participantes que cursavam o ensino médio na rede pública (54,8\%).

Quando analisamos as respostas dos alunos do ensino médio quanto ao tipo de doação vigente no Brasil, percebemos dúvidas ainda existentes. Já entre os adolescentes e adultos jovens dos cursos na área da saúde, percebemos um impacto maior com relação às mudanças legislativas, pois a maioria deles acreditava que para ser doador ainda seria preciso registrar seu desejo em documento de identidade, talvez, pelo fato da pesquisa ter sido realizada após a mudança da legislação e isso proporcionar maior discussão na imprensa falada.

Há evidências de que a informação cria resultados em curto prazo e a educação gera a médio e longo prazo, sendo assim necessária a incorporação desse tema nas bases de ensino com o intuito de criar/ sedimentar uma cultura de doação entre os estudantes que seriam também transmissores do conhecimento adquirido.

Os percentuais e os resultados apresentados quanto ao critério de doação brasileiro, levam-nos a crer que seja um fator dificultador do processo de doação, já que as famílias desconhecem o desejo de seu ente querido em ser doador; o que já fora explorado e evidenciado em outras pesquisas como sendo um dos motivos de recusa familiar para a doação. Sabe-se que, quando as famílias conhecem mais sobre doação, tendem a ser doadoras. ${ }^{6,15}$

\section{CONCLUSÃO}

Os resultados apresentados permitem sugerir que a mudança no tipo de doação de órgão e tecidos para transplante continua representando um importante fator de dúvida para estudantes do ensino médio, pois 47,0\% (363) não sabem qual é o critério para se tornarem doadores.

Por outro lado, os estudantes do ensino superior também desconhecem o critério e apresentam conhecimento inferior quando comparados aos do ensino médio, pois 50,6\% (316) não sabiam que para ser um doador era necessário comunicar a família.

A prevalência de doadores entre os estudantes em geral foi de 66,3\% (946), enquanto os não doadores representaram 23,1\% (329), 8,9\% (127) não sabiam e 1,8\% (25) não informaram (Figura 3).

Talvez isto signifique que os esforços educacionais individuais e as pequenas campanhas ou feiras de ciências no ensino médio, tenham colaborado, mesmo que em pequena proporção, para a incorporação modesta de um novo cenário.

Os resultados mostram que, apesar da mudança na lei dos transplantes, alguns princípios básicos para se construir uma educação permanente nessa área, não se desenvolveram, sugerindo que o atraso cultural de dez anos indicado por alguns juristas, após a aprovação do tipo de doação, fez-se presente na amostra estudada.

Por fim, estratégias educacionais, e não somente informativas, precisam fazer parte do currículo da educação básica brasileira, para o desenvolvimento de uma cultura de doação no país, o que subsidiaria a decisão de ser ou não doador. Para que funcionem, essas estratégias dependerão, não somente da regulamentação dos governos, mas também da vontade dos principais envolvidos nesse processo.

Figura 3. Prevalência de doadores entre os estudantes

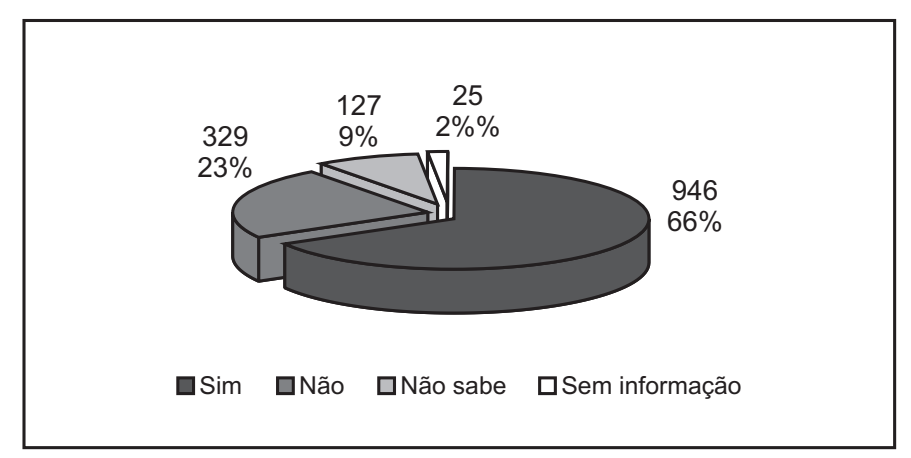

\section{ABSTRACT}

Changes in Brazi'lian law 9.434/97, by a provisory standard with a posterior alteration by the law 2.211/2001, relation to the ways of donation, transforming the donation from presumed (1997) to consented (2001), these could have collaborated, trough other elements, to the difficult of a donation culture incorporation among our country. Knowing students opinions is, therefore, a necessary condition to develop educational strategies and help to build a legislation that represents, in fact, the population morality. Purpose: to investigate the prevalence of donors of organs and tissues among high school and college students, and their knowledge about the family consent as prerogative to donation in Brazil. Methods: study of a quantitative approach, based on a comparison of similar variables from two researches about the knowledge of health college students and high school students at the last year, at São Paulo city, made in different periods, 2002/2003 and 2005/2006, respectively. Results: 47\% (363) students from high school and 50,6\% (316) from college did not know that in Brail the organ donation is consented. There are evidences of a greater percentage of not donors among college students; and the opposite happens with high school students who had a bigger percentage of donors. Conclusion: the prevalence of organ donors among students generally speaking was 66,3\% (946), while not donors were 23,1\% (329), 8,9\% (127) did not know and 1,8\% (25) did not informed. Therefore, educational strategies, and not only informative, need to be part of the Brazilian basic school curriculum, to development of a donor culture at the country, to give a subsidy to decide being or not a organ donor. These strategies will depend not only of government regulation, but also of the intentions of the principal involved on this process for its success.

Keywords: Students, Transplantation, Public Opinion, policies, Knowledge Bases. 


\section{REFERÊNCIAS}

1. Brasil. Ministério da Saúde. Secretaria de assistência à saúde: Relatório de gestão da secretaria de assistência à saúde, 2a. Ed. revisada e modificada. 2002.

2. Lei n. 9.434, de fevereiro de 1997. Dispõe sobre a remoção de órgãos, tecidos e partes do corpo humano para fins de transplante, e dá outras providências. Diário Oficial da União, Brasília; 1997. p. 2191-3.

3. Decreto $n^{\circ} 2.268$, de 30 de junho de 2007. Regulamenta a Lei $n^{\circ} 9.434$, de 4 de fevereiro de 1997, Brasília; 2001. p. 06- Edição Extra.

4. Garcia VD. Por uma política de transplante no Brasil. São PAulo: Office Editora e Publicidade Ltda; 2000.

5. Roza BA: Impacto da Legislação dos Transplantes de Órgãos sobre Médicos e Enfermeiros de uma Comunidade Universitária. [Tese de Mestrado]. São Paulo (SP): Universidade Federal de São Paulo; 2000

6. Roza BA: Efeitos do processo de doação de órgãos e tecidos em familiares:intencionalidade de uma nova doação. Tese de Doutorado apresentada à Universidade Federal de São Paulo, 2005.

7. RBT - Registro Brasileiro de Transplantes - ABTO - Ano XII - $\mathrm{n}^{\circ} 2$ - Janeiro / dezembro 2006

8. MICHAELIS: pequeno dicionário da língua portuguesa - São Paulo: Companhia Melhoramentos; 1998. termos pesquisados: Informar e educação.
9. Sanner MA: A Swedish survey of young people's views on organ donation and transplantation .Transplantation Proceedings. 2004;36:431-2

10. Spigner C, Weaver M, Cardenas V, Allen MD. Organ donation and transplantation:ethnic differences in knowledge and opinions among urban high school students. Ethn Health . 2002;7:87-101.

11. Piccoli GB, Soragna G, Putaggio S, Burdese M, Longo P, Rinaldi D, et al. Efficacy of an educational program on dialysis, renal transplantation, and organ donation on the opinions of high school students: a randomized controlled trial. Transplantation Proceedings. 2004:431-2.

12. Perón AL, Rodrigues AB, Leite DA, Lopes JL, Ceschim PC, Alter R. Conhecimento e opinião dos alunos de graduação da UNIFESP sobre doação de órgãos e transplantes no Brasil. São Paulo, 2002.

13. Silva AS, Schirmer J, Roza BA, Fujinami TI. Estudo das opiniões de estudantes do ensino médio sobre doação e transplante de órgãos e tecidos, São Paulo, 2005.

14. Silva VS; Schirmer J, Roza BA, Silva AS, Fujinami TI. Estudo das opiniões de estudantes do ensino médio sobre doação e transplante de órgãos e tecidos, São Paulo, 2006.

15. Moraes EL. A recusa familiar no processo de doação de órgãos e tecidos para transplante. 142 p. São Paulo, 2007. 